\title{
Exceptional Points in Atomic Spectra
}

\author{
Holger Cartarius, Jörg Main, and Günter Wunner \\ Institut für Theoretische Physik 1, Universität Stuttgart, 70550 Stuttgart, Germany
}

(Dated: January 29, 2018)

\begin{abstract}
We report the existence of exceptional points for the hydrogen atom in crossed magnetic and electric fields in numerical calculations. The resonances of the system are investigated and it is shown how exceptional points can be found by exploiting characteristic properties of the degeneracies, which are branch point singularities. A possibility for the observation of exceptional points in an experiment with atoms is proposed.
\end{abstract}

PACS numbers: 32.60.+i, 02.30.-f, 32.80.Fb

The appearance of the coalescence of two eigenstates, so-called exceptional points [1], in physical systems described by non-Hermitian matrices has attracted growing interest [2, 3, 4, 5, 6]. Typical systems in which such a phenomenon can occur are open quantum systems with decaying unbound states. One possibility to describe these open quantum systems are non-Hermitian Hamiltonians obtained with the complex rotation method [7]. In this case, the resonances appear as complex eigenvalues whose real and imaginary parts are connected with the energy and the resonance width, respectively. The eigenvectors have not to be orthogonal in contrast to Hermitian Hamiltonians describing bound states in quantum mechanics. In particular, it is possible that the eigenspace for two degenerate eigenvalues is only onedimensional, i.e., there is only one linear independent eigenvector. If the system of interest depends on a complex parameter $\lambda$ (or two real parameters), a branch point singularity of two eigenstates can appear at critical parameter values $\lambda_{c}$, which are called exceptional points. Exceptional points have been discovered in a broad variety of physical systems. Among them are acoustical systems [8], atoms in optical lattices [4, 9], and complex atoms in laser fields [10]. Detailed experiments have been carried out with resonances in microwave cavities [5, 6, 11]. However, up to now exceptional points have not been found in atoms in static external fields. The main reason is that there is only one parameter in the cases studied most intensely, viz. atoms either in a magnetic or in an electric field. For the occurrence of exceptional points, the parameter space has to be at least two-dimensional, i.e., at least two real parameters are required, which can be represented by crossed magnetic and electric fields. Atoms in static external magnetic and electric fields are fundamental physical systems. As real quantum systems they are accessible both with experimental and theoretical methods and have been used for comparisons with semiclassical theories [12, 13, 14]. They are ideally suited to study the influence of exceptional points on quantum systems. E.g., the occurrence of phenomena like Ericson fluctuations in photoionization spectra has been demonstrated both in numerical studies [15, 16] and experiments [17].
In this Letter, we investigate numerically the resonances of the hydrogen atom in static magnetic and electric fields and report the first detection of exceptional points in this system. The confirmation of the existence of exceptional points supplements the richness of phenomena which have been found in the spectra of atoms in static external fields. Furthermore, we propose a method which can be used to verify the existence of exceptional points in experiments.

Exceptional points can occur in systems which are described by a non-Hermitian matrix and depend on one complex parameter or two real parameters. At critical parameter values $\lambda_{c}$, the eigenvalues and eigenvectors of the matrix pass through branch point singularities [3, 4, 18]. This can easily be understood by studying the two-dimensional matrix [1]

$$
\boldsymbol{M}(\lambda)=\left(\begin{array}{rr}
1 & \lambda \\
\lambda & -1
\end{array}\right)
$$

with the complex parameter $\lambda$. The eigenvalues $\epsilon_{1}=$ $\sqrt{1+\lambda^{2}}$ and $\epsilon_{2}=-\sqrt{1+\lambda^{2}}$ are two branches of one analytic function in $\lambda$. An exceptional point occurs, e.g., for $\lambda=\mathrm{i}$, where the eigenvectors of the two degenerate eigenvalues coalesce and the only linearly independent eigenvector reads $\boldsymbol{x}(\lambda=\mathrm{i})=(1, \mathrm{i})^{T}$. The branch point singularity leads to a characteristic behavior of the corresponding eigenvalues under changes of the parameters. If one chooses a closed loop in the parameter space and calculates the eigenvalues for a set of parameter values on this loop, in general, the eigenvalues also traverse a closed curve. However, when an exceptional point is encircled, two of the eigenvalues do not traverse a closed loop. The two eigenvalues which degenerate at the exceptional point are permuted during the traversal of the circle in parameter space [1]. This can be seen by plotting the paths of the two eigenvalues in the complex energy plane. After one circle, the first eigenvalue will travel to the starting point of the second and vice versa, as illustrated in Fig. 1. As a consequence, the path of one eigenvalue is not closed, if one traversal of the loop in parameter space is performed. But the path is closed if the parameter space loop is traversed twice.

As one would expect, the eigenstates of the system are 

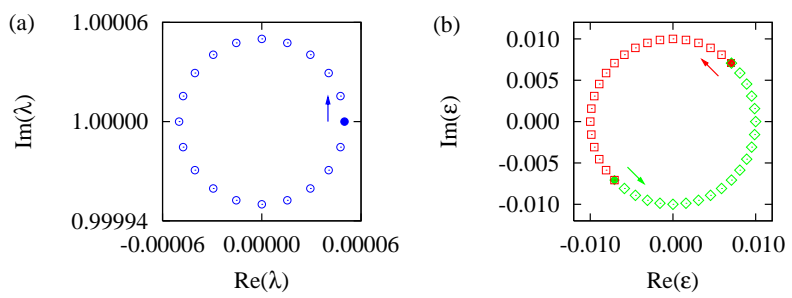

FIG. 1: (Color online) (a) Circle in parameter space $\lambda$ with the exceptional point $\lambda_{c}=\mathrm{i}$ as center point for the simple model (1). (b) Eigenvalues $\epsilon_{1,2}$ calculated for the parameter values from (a) indicated by squares and diamonds, respectively. In this special case, each of the two eigenvalues traverses a semicircle. In (a) and (b), the filled symbols represent the first parameter value $\lambda_{0}$ and the corresponding eigenvalues $\epsilon_{1,2}\left(\lambda_{0}\right)$, respectively. The arrows point in the direction of progression.

permuted in the same way as the corresponding eigenvalues [1]. Additionally, after each circle around the exceptional point, one of the two eigenvectors changes its sign [19], e.g., $\left[\Psi_{1}, \Psi_{2}\right] \stackrel{\text { circle }}{\rightarrow}\left[\Psi_{2},-\Psi_{1}\right]$. This phase change continues with every additional loop. Therefore, four circles are required to return to the initial states.

In this Letter we investigate exceptional points in the hydrogen atom in static external fields. The Hamiltonian in atomic units without relativistic corrections and finite nuclear mass effects [20] reads

$$
H=\frac{1}{2} p^{2}-\frac{1}{r}+\frac{1}{2} \gamma L_{z}+\frac{1}{8} \gamma^{2}\left(x^{2}+y^{2}\right)+f x,
$$

where $L_{z}$ is the $z$ component of the angular momentum, and $\gamma$ and $f$ are the field strengths of the magnetic and electric fields oriented along the $z$ - and $x$-axis, respectively. The only constants of motion are the energy and the parity with respect to the $(z=0)$-plane. Here, we concentrate on states with even $z$-parity. One possibility to calculate the resonances of the system is the complex rotation method [7, 21]. The coordinates of the system $\boldsymbol{r}$ are replaced with the complex rotated ones $\boldsymbol{r} e^{\mathrm{i} \vartheta}$ in the Hamiltonian and wave functions. The transformation leads to a complex symmetric matrix representation of the Hamiltonian. Introducing dilated semiparabolic coordinates (see, e.g., 16]) leads to a generalized eigenvalue problem of the form

$$
\boldsymbol{A}(\gamma, f) \Psi=\kappa \boldsymbol{B} \Psi
$$

with a complex symmetric matrix $\boldsymbol{A}(\gamma, f)$, a real symmetric matrix $\boldsymbol{B}$, and the generalized eigenvalue $\kappa=$ $-\left(1+2 b^{4} E\right)$, where $E$ is the energy and $b$ a complexvalued dilation parameter, which includes the complex rotation. Above the ionization threshold, resonances are uncovered as complex energies $E$. The computation of the eigenvalues was performed by applying the ARPACK library 22] to matrices with dimensions of about 10,000 to 12,300 . The algorithm uses the implicitly restarted Arnoldi method and solves large scale sparse eigenvalue problems efficiently, even for non-Hermitian matrices. In general, but not at the exceptional points, the eigenvectors of the resonances can be normalized such that $\left\langle\Psi_{i}|\boldsymbol{B}| \Psi_{j}\right\rangle=\delta_{i j}$. The external fields $\gamma$ and $f$ are the two external parameters which determine the eigenstates. Exceptional points do exist in atomic spectra if the fields can be chosen in such a way that a coalescence of two states occurs. The crossed field hydrogen system fulfills all necessary conditions for the appearance of exceptional points, however, one has to find them in the spectrum to prove their existence.

In practice, it is very difficult to look for exceptional points by searching for degeneracies of two complex eigenvalues. The variation of the parameter values $\gamma$ and $f$ does not lead to clear indications for degeneracies, and, it is not known in advance which parameter values are a good choice for starting the search. However, the method of encircling a point in the $(\gamma, f)$-plane and searching for eigenvalues which are permuted has been very successful. A good choice for such a closed loop is a "circle" in the parameter space of the two field strengths with a radius $a<1$ chosen relative to the center $\left(\gamma_{0}, f_{0}\right)$ :

$$
\begin{aligned}
& \gamma(\varphi)=\gamma_{0}(1+a \cos (\varphi)), \\
& f(\varphi)=f_{0}(1+a \sin (\varphi)) .
\end{aligned}
$$

The phase change of one of the eigenfunctions after a circle around an exceptional point can be seen as a sign change of an arbitrary matrix element $p_{12}=\left\langle\Psi_{1}|M| \Psi_{2}\right\rangle$. We chose $M=1$, i.e.,

$$
p_{i j}=\left\langle\Psi_{i} \mid \Psi_{j}\right\rangle,
$$

which can be obtained easily in our calculations and is not diagonal for the eigenstates which fulfill the orthogonality relation $\left\langle\Psi_{i}|\boldsymbol{B}| \Psi_{j}\right\rangle=\delta_{i j}$ mentioned above.

With the method described, exceptional points have been found for the first time in the spectrum of the hydrogen atom in static external fields. Fig. 2 (a) shows a typical result obtained in a numerical calculation. The squares and the diamonds represent one of the eigenvalues at different field strengths, respectively. In this example, using 20 steps on the circle in parameter space has been sufficient to obtain a clear signature of the branch point singularity. The "radius" of the circle according to Eq. (4) was $a=0.01$. This value is sufficiently large to have an exceptional point inside the circular area with a high probability and is small enough to track the paths of the eigenvalues with a low number of calculations. Fig. 2 (b) shows the position of the degenerate eigenvalues (marked with an arrow) in the complex energy plane among the resonances in their vicinity. For the loop shown in Fig. 2 (a), the matrix element $p$ from Eq. (5) was calculated. The phase of the complex value, 

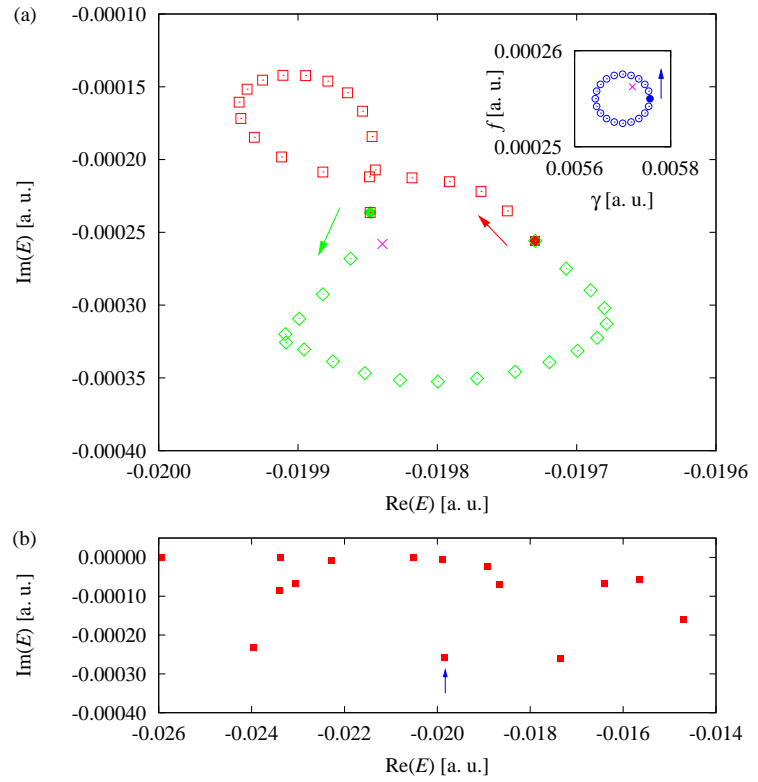

FIG. 2: (Color online) (a) Paths of the two eigenvalues which degenerate at the exceptional point in the complex energy plane. The squares and the diamonds represent one of the eigenvalues, respectively. Each point of one eigenvalue belongs to a different set of parameters. The path in the field strength parameter space is a circle defined in Eq. (4) with $a=0.01$ (see inset). The first set of parameters and the corresponding eigenvalues are represented by filled symbols. The arrows indicate the direction of progression. The cross marks the position of the exceptional point in parameter space and the corresponding complex energy of the degenerate resonances. (b) Resonances in the complex energy plane for the parameter values $\gamma=0.00572$ and $f=0.000256$ (atomic units) at the exceptional point. The position of the two degenerate eigenvalues is marked with an arrow.

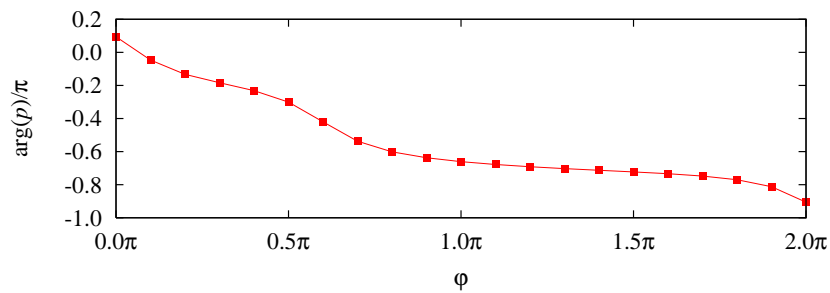

FIG. 3: (Color online) Phase of the complex matrix element $p$ defined in Eq. (5). It changes its value by $\pi$ during the traversal of the circle in parameter space. The result corresponds to the expected sign change.

plotted in Fig. 3. changes its value by $\pi$. This clearly indicates a sign change for one of the two eigenstates 19] as mentioned above.

Examples for parameter values of exceptional points and the position of the degenerate eigenvalues in the complex energy plane are given in Table I The values were obtained by minimizing the distance of two eigenvalues which indicated a branch point singularity. The
TABLE I: Examples for exceptional points in the spectrum of the hydrogen atom in crossed magnetic $(\gamma)$ and electric $(f)$ fields. All values in atomic units.

\begin{tabular}{ccll}
\hline \hline$\gamma$ & $f$ & \multicolumn{1}{c}{$\operatorname{Re}(E)$} & \multicolumn{1}{c}{$\operatorname{Im}(E)$} \\
\hline 0.00537 & 0.000214 & -0.01884 & -0.0000679 \\
0.00572 & 0.000256 & -0.01984 & -0.000258 \\
0.00611 & 0.000256 & -0.01593 & -0.00024 \\
0.00615 & 0.000265 & -0.0158 & -0.000374 \\
\hline \hline
\end{tabular}

degeneracy of the two eigenvalues, the existence of only one eigenvector, and the permutation of eigenvalues were used to identify the exceptional points.

In experiments, the complex eigenvalues cannot be obtained directly. But it is possible to measure the photoionization cross section and to extract the energy (real part of a complex eigenvalue) and width (imaginary part) of the resonances. The cross section can be written in the form 23]

$$
\sigma(E)=4 \pi \alpha\left(E-E_{0}\right) \operatorname{Im}\left(\sum_{j} \frac{\left\langle\Psi_{0}^{(\vartheta)}|D| \Psi_{j}^{(\vartheta)}\right\rangle^{2}}{E_{j}-E}\right),
$$

where $\Psi_{0}^{(\vartheta)}$ is the initial state with energy $E_{0}$, which is supposed to be known. The final state with complex energy $E_{j}$ is represented by $\Psi_{j}^{(\vartheta)}, D$ is the dipole operator for a given polarization, and $\alpha$ is the fine-structure constant. The superscript $\vartheta$ on the initial and final states indicates the angle of the complex rotation used in the calculation, however, it is important to note that the cross section is independent of that angle in converged spectra. The Fourier transform $c(t)$ (i.e., the time signal) of (6) has the form $c(t)=\sum_{j} a_{j} \exp \left(\mathrm{i} E_{j} t\right)$ and, therefore, the complex energies $E_{j}$ can be obtained using the harmonic inversion method [24] with a high precision. In this method, the Fourier transform of the profiles in the photoionization cross section is used to extract their complex amplitudes $a_{j}$ and energies $E_{j}$ by solving the nonlinear set of equations

$$
c_{n}=\sum_{j} a_{j} \exp \left(\mathrm{i} E_{j} t\right), \quad n=0,1, \ldots, 2 K-1
$$

for $K$ values $c_{n}=c\left(t_{n}\right)$ on an equidistant grid.

With this knowledge, a search for exceptional points in an experiment starts with the measurement of the photoionization cross section for different field strengths, which are located on a closed loop in the $(\gamma, f)$-space, e.g., on a circle of type (4). For each measurement, one obtains a spectrum which is used to extract the complex energies of the resonances. The energy values are drawn in a diagram. After these steps, the same method which is used to search for branch point singularities in numerical calculations can be applied to the experimental results. The diagrams can be used to look for the characteristic open curves of single eigenvalues. Fig. 目 (a) 
(a)
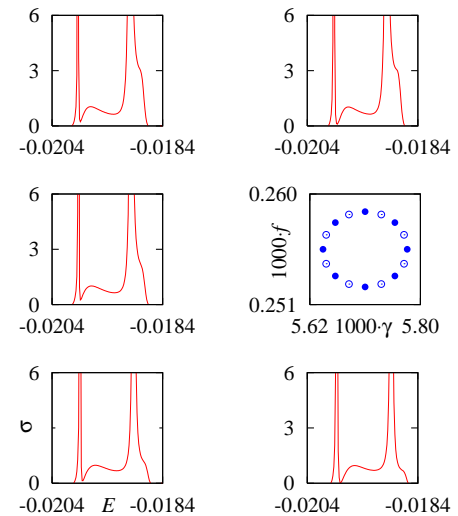

(b)

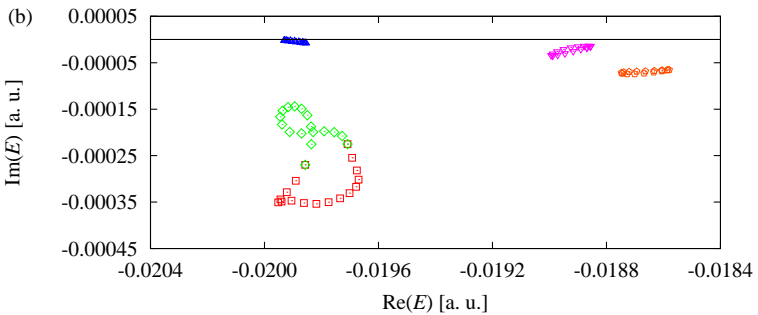

FIG. 4: (Color online) (a) Photoionization cross section of the energy range in which two eigenvalues connected with an exceptional point appear. The cross section is shown for eight different pairs of parameter values $\gamma$ and $f$ being located on a circle around the exceptional point. All values in atomic units. (b) Complex energy eigenvalues extracted from the cross sections with the harmonic inversion method. Each eigenvalue is drawn with a different symbol. Altogether, 16 pairs of parameter values were used. In (a), every second cross section is shown. The signature of a branch point singularity connected with the two eigenvalues labeled with squares and diamonds, respectively, is clearly visible.

shows an example of a typical result for the photoionization cross section for different field strengths which are located on a closed loop in the parameter space. The loop encircles an exceptional point. Although not experimental but theoretical photoionization spectra have been used as input for the calculations, it is obvious that it is possible to extract the complex energies from a typical cross section as it is obtained in an experiment. In Fig. 4 (b) one can see the paths of complex eigenvalues extracted from the spectra. In our example, 16 cross sections were used. This number is sufficient to give a clear indication of an exceptional point as shown in Fig. 4 (b). The eight spectra in Fig. 4 (a) look rather similar. Note that the deviations become more pronounced when the parameter space radius is increased. This may be helpful for the analysis of experimental spectra with noise.

In summary, we have found the first exceptional points in numerical spectra of an atom in static external fields. The branch point singularities can be detected by the permutation of two eigenvalues when an exceptional point is encircled in parameter space. Further properties of the branch point singularities can be used to verify their existence. With the harmonic inversion method, it is possible to extract the complex eigenvalues of resonances from experimental photoionization cross sections and to detect exceptional points in experimental data. This opens the way for the experimental observation of exceptional points in atomic spectra.

J.M. acknowledges stimulating discussions with W. D. Heiss. This work was supported by DFG. H.C. is grateful for the support from the LGFG of the Land BadenWürttemberg.

[1] T. Kato, Perturbation theory for linear operators (Springer, Berlin, 1966).

[2] W. D. Heiss, Phys. Rev. E 61, 929 (2000).

[3] W. D. Heiss and A. L. Sannino, J. Phys. A 23, 1167 (1990).

[4] M. V. Berry and D. H. J. O'Dell, J. Phys. A 31, 2093 (1998).

[5] C. Dembowski, H.-D. Gräf, H. L. Harney, A. Heine, W. D. Heiss, H. Rehfeld, and A. Richter, Phys. Rev. Lett. 86, 787 (2001).

[6] C. Dembowski, B. Dietz, H.-D. Gräf, H. L. Harney, A. Heine, W. D. Heiss, and A. Richter, Phys. Rev. Lett. 90, 034101 (2003).

[7] Y. K. Ho, Phys. Rep. 99, 1 (1983).

[8] A. L. Shuvalov and N. H. Scott, Acta Mech. 140, 1 (2000).

[9] M. K. Oberthaler, R. Abfalterer, S. Bernet, J. Schmiedmayer, and A. Zeilinger, Phys. Rev. Lett. 77, 4980 (1996).

[10] O. Latinne, N. J. Kylstra, M. Dörr, J. Purvis, M. TeraoDunseath, C. J. Joachain, P. G. Burke, and C. J. Noble, Phys. Rev. Lett. 74, 46 (1995).

[11] M. Philipp, P. v. Brentano, G. Pascovici, and A. Richter, Phys. Rev. E 62, 1922 (2000).

[12] S. Freund, R. Ubert, E. Flöthmann, K. H. Welge, D. M. Wang, and J. B. Delos, Phys. Rev. A 65, 053408 (2002).

[13] J. Rao, D. Delande, and K. T. Taylor, J. Phys. B 34, L391 (2001).

[14] T. Bartsch, J. Main, and G. Wunner, Phys. Rev. A 67, 063410 and 063411 (2003).

[15] J. Main and G. Wunner, Phys. Rev. Lett. 69, 586 (1992).

[16] J. Main and G. Wunner, J. Phys. B 27, 2835 (1994).

[17] G. Stania and H. Walther, Phys. Rev. Lett. 95, 194101 (2005).

[18] W. D. Heiss and A. L. Sannino, Phys. Rev. A 43, 4159 (1991).

[19] W. D. Heiss, Eur. Phys. J. D 7, 1 (1999).

[20] P. Schmelcher and L. S. Cederbaum, Chem. Phys. Lett. 208, 548 (1993).

[21] D. Delande, A. Bommier, and J. C. Gay, Phys. Rev. Lett. 66, 141 (1991).

[22] R. B. Lehoucq, D. C. Sorensen, and C. Yang, ARPACK Users' Guide (Siam, Philadelphia, 1998).

[23] T. N. Rescigno and V. McKoy, Phys. Rev. A 12, 522 (1975).

[24] Dž. Belkić, P. A. Dando, J. Main, and H. S. Taylor, J. Chem. Phys. 113, 6542 (2000). 